УДК 517.521

\author{
N. Subramanian, A. Esi
}

\title{
WIJSMAN ROUGH CONVERGENCE OF TRIPLE SEQUENCES
}

\author{
N. Subramanian, A. Esi. Wijsman rough convergence of triple sequences, Mat. Stud. 48 (2017), \\ $171-179$.
}

In this paper we define and study Wijsman rough convergence of triple sequences, the set of Wijsman rough limit points of a triple sequence. Also investigate the relations between the set of cluster points and the set of Wijsman rough limit points of a triple sequence.

10.15330/ms.48.2.171-179

1. Introduction. Let $(X,\|\cdot\|)$ be a normed linear space. A point $x_{*} \in X$ is said to be an $r$-limit point of a sequence $x=\left(x_{i}\right)$ in $(X,\|\cdot\|)$ if $\limsup _{i \rightarrow \infty}\left\|x_{i}-x_{*}\right\| \leq r(r \geq 0)$. Denote by $\operatorname{LIM}_{x}^{r}$ the set of all $r$-limit points of $\left(x_{i}\right)$. The idea of rough convergence was first introduced by $\mathrm{Phu}[9-11]$ in finite dimensional normed spaces. He showed that the set $\mathrm{LIM}_{x}^{r}$ is bounded, closed and convex; and introduced the notion of a rough Cauchy sequence. He also investigated the relations between the rough convergence and other convergence types and the dependence of $\operatorname{LIM}_{x}^{r}$ on the roughness of degree $r$.

Aytar [1] studied of the rough statistical convergence and defined the set of rough statistical limit points of a sequence and obtained two statistical convergence criteria associated with this set and prove that this set is closed and convex. Also, Aytar [2] established that the $r$-limit set of the sequence is equal to the intersection of these sets and that $r$-core of the sequence is equal to the union of these sets. Dündar and Cakan [8] investigated of rough ideal convergence and defined the set of rough ideal limit points of a sequence.

Let $(X, d)$ be a metric space. For any non empty closed subsets $A$ and $A_{m n k} \subset X$, $(m, n, k) \in \mathbb{N}^{3}$, we say that the triple sequence $\left(A_{m n k}\right)$ is Wijsman statistically convergent to $A$ if the triple sequence $\left(d\left(x, A_{m n k}\right)\right)$ is statistically convergent to $d(x, A)$, i.e., for $\varepsilon>0$ and for each $x \in X$

$$
\lim _{r, s, t \rightarrow \infty} \frac{1}{r s t}\left|\left\{(m, n, k), m \leq r, n \leq s, k \leq t:\left|d\left(x, A_{m n k}\right)-d(x, A)\right| \geq \varepsilon\right\}\right|=0 .
$$

In this case, we write $\mathrm{St-} \lim _{m, n, k \rightarrow \infty} A_{m n k}=A$ or $A_{m n k} \longrightarrow A(W S)$. The triple sequence $\left(A_{m n k}\right)$ is bounded if $\sup _{m n k} d\left(x, A_{m n k}\right)<\infty$ for each $x \in X$.

A triple sequence (real or complex) can be defined as a function $x: \mathbb{N}^{3} \rightarrow \mathbb{R}(\mathbb{C})$, where $\mathbb{N}, \mathbb{R}$ and $\mathbb{C}$ denote the set of natural numbers, real numbers and complex numbers respectively. The different types of notions of triple sequence was introduced and investigated at the initial by Sahiner et al. [12,13], Esi et al. [3-5], Datta et al. [6], Subramanian et al. [14], Debnath et al. [7] and many others.

A triple sequence $x=\left(x_{m n k}\right)$ is said to be triple analytic if

2010 Mathematics Subject Classification: 40A05, 40C99.

Keywords: triple sequences; Wijsman rough convergence; rough limit points.

(C) N. Subramanian, A. Esi, 2017 


$$
\sup _{m, n, k}\left|x_{m n k}\right|^{\frac{1}{m+n+k}}<\infty \text {. }
$$

The space of all triple analytic sequences are usually denoted by $\Lambda^{3}$.

In this paper, we introduce the notion of Wijsman rough convergence and the set of Wijsman rough limit points of a triple sequence and obtained two Wijsman rough convergence criteria associated with this set, also the relations between the set of accumulation points and the set of Wijsman rough limit points of a triple sequence.

2. Definitions and Preliminaries. Consider a triple sequence $x=\left(x_{m n k}\right)$. The following definitions are introduced.

Definition 1. A triple sequence $x=\left(x_{m n k}\right)$ is said to be Wijsman rough convergent ( $r$ convergent) to $A$, denoted as $A_{m n k} \rightarrow^{r} A\left(d\left(x, A_{m n k}\right) \rightarrow^{r} d(x, A)\right)$, provided that

$$
\forall \varepsilon>0, \exists i_{\varepsilon} \in \mathbb{N}: m, n, k \geq i_{\varepsilon} \Longrightarrow\left|d\left(x, A_{m n k}\right)-d(x, A)\right|<r+\varepsilon,
$$

or equivalently, if

$$
\lim \sup \left|d\left(x, A_{m n k}\right)-d(x, A)\right| \leq r .
$$

Here $r$ is called the Wijsman roughness of degree. If we take $r=0$, then we obtain the ordinary Wijsman convergence of a triple sequence.

Definition 2. If (1) holds, $d(x, A)$ is called Wijsman rough limit point of $\left(d\left(x, A_{m n k}\right)\right)$, the Wijsman rough limit set (or shortly: $r$-limit set) of the triple sequence $\left(d\left(x, A_{m n k}\right)\right)$ is defined by

$$
\operatorname{LIM}^{r}\left(d\left(x, A_{m n k}\right)\right):=\left\{d(x, A) \in \mathbb{R}: d\left(x, A_{m n k}\right) \rightarrow^{r} d(x, A)\right\} .
$$

Definition 3. A triple sequence $x=\left(x_{m n k}\right)$ is said to be Wijsman $r$-convergent if $\operatorname{LIM}^{r}\left(d\left(x, A_{m n k}\right)\right) \neq \varnothing$. In this case, $r$ is called the Wijsman convergence degree of the triple sequence $\left(d\left(x, A_{m n k}\right)\right)$. For $r=0$, we get the ordinary Wijsman convergence.

Remark 1. A triple sequence $y=\left(y_{m n k}\right)$ is Wijsman convergent and cannot be calculated exactly; the approximated triple sequence $\left(d\left(x, A_{m n k}\right)\right)$ satisfying $\left|d\left(x, A_{m n k}\right)-d\left(y, A_{m n k}\right)\right|$ $\leq r$ for all $m, n, k$ where $r>0$ is an upper bound of approximation error. Then, the triple sequence $\left(d\left(x, A_{m n k}\right)\right)$ is no more Wijsman convergent; but

$$
\begin{aligned}
\left|d\left(x, A_{m n k}\right)-d(x, A)\right| \leq & \left|d\left(x, A_{m n k}\right)-d\left(y, A_{m n k}\right)\right|+\left|d\left(y, A_{m n k}\right)-d(x, A)\right| \leq \\
& \leq r+\left|d\left(y, A_{m n k}\right)-d(x, A)\right|
\end{aligned}
$$

implies that it is $r$-convergent in the sense of (1) in the case $\left|d\left(y, A_{m n k}\right)-d(x, A)\right| \rightarrow 0$. The $r$-limit set of a triple sequence is not unique for the Wijsman roughness degree $r>0$.

Definition 4. A point $d\left(x, A_{m n k}\right) \in X$ is called a Wijsman cluster point or accumulation point of a triple sequence $\left(x_{m n k}\right)$ if, for every neighbourhood $V$ of $d\left(x, A_{m n k}\right)$, there are infinitely many natural numbers $(m, n, k)$ such that $d\left(x, A_{m n k}\right) \in V$.

If the space is Fréchet-Urysohn, this is equivalent to the assertion that $x$ is a limit of some subsequence of the sequence $\left(d\left(x, A_{m n k}\right)\right)$.

\section{Main Results.}


Theorem 1. A Wijsman triple sequence $x=\left(x_{m n k}\right)$ is analytic if and only if there exists an $r \geq 0$ such that $\operatorname{LIM}^{r}\left(d\left(x, A_{m n k}\right)\right) \neq \varnothing$. For all $r \geq 0$, a Wijsman triple analytic sequence $d\left(x, A_{m n k}\right)$ always contains a subsequence $\left(d\left(x, A_{m_{i} n_{j} k_{\ell}}\right)\right)$ with $\operatorname{LIM}^{r}\left(d\left(x, A_{m_{i} n_{j} k_{\ell}}\right)\right) \neq \varnothing$.

Proof. If $s=\sup \left\{\left|d\left(x, A_{m n k}\right)\right|^{1 / m+n+k}:(m, n, k) \in \mathbb{N}^{3}\right\}<\infty$ then $\operatorname{LIM}^{s}\left(d\left(x, A_{m n k}\right)\right)$ contains the origin of $X$. On the other hand, if $\operatorname{LIM}^{r}\left(d\left(x, A_{m n k}\right)\right) \neq \varnothing$ for some $r \geq 0$ then all but finite elements $d\left(x, A_{m n k}\right)$ are contained in some ball with any radius greater than $r$. Hence, the triple sequence $\left(d\left(x, A_{m n k}\right)\right)$ is analytic.

As $\left(d\left(x, A_{m n k}\right)\right)$ is a Wijsman triple analytic seqeuence in a three dimensional metric space, it has convergent subsequence $\left(d\left(x, A_{m_{i} n_{j} k_{\ell}}\right)\right)$. Let $d(x, A)$ be its limit point then $\operatorname{LIM}^{r}\left(d\left(x, A_{m n k}\right)\right)=\bar{B}_{r}(d(x, A))$ and for $r>0$,

$$
\operatorname{LIM}^{r}\left(d\left(x, A_{m_{i} n_{j} k_{\ell}}\right)\right)=\left\{d\left(x, A_{m_{i} n_{j} k_{\ell}}\right):\left|d(x, A)-d\left(x, A_{m_{i} n_{j} k_{\ell}}\right)\right| \leq r\right\} \neq \varnothing .
$$

Theorem 2. If $\left(d\left(x, A_{m n k}\right)\right)$ is a subsequence of Wijsman triple sequence $\left(d\left(x, A_{m n k}\right)\right)$, then $\operatorname{LIM}^{r}\left(d\left(x, A_{m n k}\right)\right) \subseteq \operatorname{LIM}^{r}\left(d\left(x, A_{m n k}\right)\right)^{\prime}$.

Theorem 3. Suppose $r_{1} \geq 0$ and $r_{2}>0$. A triple sequence $x=\left(x_{m n k}\right)$ is $r_{1}+r_{2}$-convergent to $d(x, A)$ if and only if there exist a triple sequence $y=\left(y_{m n k}\right)$ such that

$$
d\left(y, A_{m n k}\right) \rightarrow^{r_{1}} d(y, A) \text { and }\left|d\left(x, A_{m n k}\right)-d\left(y, A_{m n k}\right)\right| \leq r_{2} \text {, for each }(m, n, k) \in \mathbb{N}^{3} .
$$

Proof. Assume that $(4) d\left(y, A_{m n k}\right) \rightarrow^{r_{1}} d(y, A)$ means that $\forall \varepsilon>0 \exists m_{\varepsilon} n_{\varepsilon} k_{\varepsilon}$ :

$$
\left|d\left(y, A_{m n k}\right)-d(y, A)\right| \leq r_{1}+\varepsilon \text { if } m \geq m_{\varepsilon}, n \geq n_{\varepsilon}, k \geq k_{\varepsilon} .
$$

Since $\left|d\left(x, A_{m n k}\right)-d\left(y, A_{m n k}\right)\right| \leq r_{2}$, this yields immediately

$$
\begin{gathered}
\left|d\left(x, A_{m n k}\right)-d(x, A)\right| \leq\left|d\left(x, A_{m n k}\right)-d\left(y, A_{m n k}\right)\right|+\left|d\left(y, A_{m n k}\right)-d(y, A)\right|<r_{1}+r_{2}+\varepsilon \\
\text { for } m \geq m_{\varepsilon}, n \geq n_{\varepsilon}, k \geq k_{\varepsilon} .
\end{gathered}
$$

Hence $\left(d\left(x, A_{m n k}\right)\right) \rightarrow^{r_{1}+r_{2}} l$.

Assume now $\left(d\left(x, A_{m n k}\right)\right) \rightarrow^{r_{1}+r_{2}} d(x, A)$ with

$$
d\left(y, A_{m n k}\right)= \begin{cases}d(y, A), & \text { if }\left|d\left(x, A_{m n k}\right)-d(x, A)\right| \leq r_{2} ; \\ d\left(x, A_{m n k}\right)+r_{2}\left(\frac{d(x, A)-d\left(x, A_{m n k}\right)}{\left|d\left(x, A_{m n k}\right)-d(x, A)\right|}\right), & \text { if }\left|d\left(x, A_{m n k}\right)-d(x, A)\right|>r_{2},\end{cases}
$$

we have

$$
\begin{aligned}
& \left|d\left(y, A_{m n k}\right)-d(y, A)\right|= \\
& =\left\{\begin{array}{l}
\left|d\left(y, A_{m n k}\right)-d\left(y, A_{m n k}\right)\right|, \quad \text { if }\left|d\left(x, A_{m n k}\right)-d(x, A)\right| \leq r_{2} ; \\
\left|d\left(x, A_{m n k}\right)-d(x, A)\right|+r_{2}\left(\frac{|d(x, A)-d(x, A)|-\left|d\left(x, A_{m n k}\right)-d(x, A)\right|}{\left|d\left(x, A_{m n k}\right)-d(x, A)\right|}\right), \\
\text { if }\left|d\left(x, A_{m n k}\right)-d(x, A)\right|>r_{2},
\end{array}\right. \\
& \left|d\left(y, A_{m n k}\right)-d(y, A)\right|= \\
& = \begin{cases}0, & \text { if }\left|d\left(x, A_{m n k}\right)-d(x, A)\right| \leq r_{2} ; \\
\left|d\left(x, A_{m n k}\right)-d(x, A)\right|-r_{2}\left(\frac{\left|d\left(x, A_{m n k}\right)-d(x, A)\right|}{\left|d\left(x, A_{m n k}\right)-d(x, A)\right|}\right), & \text { if }\left|x_{m n k}-l\right|>r_{2},\end{cases} \\
& \left|d\left(y, A_{m n k}\right)-d\left(y, A_{m n k}\right)\right|= \\
& = \begin{cases}0, & \text { if }\left|d\left(x, A_{m n k}\right)-d(x, A)\right| \leq r_{2} ; \\
\left|d\left(x, A_{m n k}\right)-d(x, A)\right|-r_{2}, & \text { if }\left|d\left(x, A_{m n k}\right)-d(x, A)\right| \leq r_{2} .\end{cases}
\end{aligned}
$$


We have $\left|d\left(y, A_{m n k}\right)-d(x, A)\right| \geq\left|d\left(x, A_{m n k}\right)-d(x, A)\right|-r_{2} \Longrightarrow$ $\left|d\left(x, A_{m n k}\right)-d(x, A)-d\left(y, A_{m n k}\right)+d(y, A)\right| \leq r_{2}$

$$
\left|d\left(x, A_{m n k}\right)-d\left(y, A_{m n k}\right)\right| \leq r_{2} \text { for }(m, n, k) \in \mathbb{N}^{3} .
$$

we have, $d(x, A) \in \mathrm{LIM}^{r_{1}+r_{2}} d\left(x, A_{m n k}\right) \Longrightarrow \lim \sup \left|d\left(x, A_{m n k}\right)-d(x, A)\right| \leq r_{1}+r_{2}$, and therefore $\lim \sup \left|d\left(y, A_{m n k}\right)-d(y, A)\right| \leq r_{1}, \Longrightarrow\left(d\left(y, A_{m n k}\right)\right) \rightarrow^{r_{1}} d(y, A)$.

Note. If $r_{1}=0$ and $r_{2}=r>0$, then $\left(d\left(x, A_{m n k}\right)\right) \rightarrow^{r} d(x, A) \Longrightarrow\left(d\left(y, A_{m n k}\right)\right) \rightarrow d(y, A)$ and $\left|d\left(x, A_{m n k}\right)-d\left(y, A_{m n k}\right)\right| \leq r,(m, n, k) \in \mathbb{N}^{3}$.

For $x=\left[\begin{array}{ccc}x_{11} & x_{12} & \cdots x_{1 n} \\ x_{21} & x_{22} & \cdots x_{2 n} \\ \vdots & & \end{array}\right]$ denote $[x]=\left[\begin{array}{ccc}{\left[x_{11}\right]} & {\left[x_{12}\right]} & \cdots\left[x_{1 n}\right] \\ {\left[x_{21}\right]} & {\left[x_{22}\right]} & \cdots\left[x_{2 n}\right] \\ \vdots & & \end{array}\right]$, where $[\alpha]$ is the integer part of the real number $\alpha$.

Theorem 4. We assume that $\left(d\left(x, A_{m n k}\right)\right) \rightarrow d(x, A)$.

(a) If $|.,$.$| is the maximum metric then d(x, A) \in \operatorname{LIM}^{3}\left[d\left(x, A_{m n k}\right)\right]$ and

$$
\operatorname{LIM}^{1.5}\left[d\left(x, A_{m n k}\right)\right] \neq \varnothing .
$$

(b) If $|.,$.$| is the Euclidean metric then d(x, A) \in \operatorname{LIM}^{\sqrt{u v w} \times 3}\left[d\left(x, A_{m n k}\right)\right]$ and

$$
\operatorname{LIM}^{\sqrt{u v w} \times 1.5}\left[d\left(x, A_{m n k}\right)\right] \neq \varnothing .
$$

Since $0 \leq d\left(x, A_{m_{i} n_{j} k_{\ell}}\right)-\left[d\left(x, A_{m_{p} n_{q} k_{r}}\right)\right]<3$ for all $(m, n, k) \in \mathbb{N}^{3}$ and $p \in(1,2, \ldots, u)$, $q \in(1,2, \ldots, v)$ and $r \in(1,2, \ldots, w)$, we have

$$
\left|d\left(x, A_{m n k}\right)-\left[d\left(x, A_{m n k}\right)\right]\right|= \begin{cases}3, & \text { if }|., .| \text { is the maximal matrix; } \\ \sqrt{u v w}, & \text { if }|., .| \text { is the Euclidean metric. }\end{cases}
$$

Let $\tilde{l}=[l]-=\left[\begin{array}{cccc}1.5 & 1.5 & \cdots & 1.5 \\ 1.5 & 1.5 & \cdots & 1.5 \\ \vdots & \vdots & \ddots & \vdots\end{array}\right]$

Since $d\left(x, A_{m n k}\right) \rightarrow d(x, A)$, there exists an $\left(m_{*}, n_{*}, k_{*}\right)$ such that $\left[d\left(x, A_{p q r}\right)\right]-3 \leq$ $d\left(x, A_{m_{p} n_{q} k_{r}}\right)-\left[d\left(x, A_{p q r}\right)\right]+3$ for $(m, n, k) \geq\left(m_{*}, n_{*}, k_{*}\right)$ and $p \in(1,2,3, \ldots, u), q \in$ $(1,2,3, \ldots, v)$ and $r \in(1,2,3, \ldots, w)$, which yields

$$
\left[d\left(x, A_{1_{p} 1_{q} 1_{r}}\right)\right] \in\left\{\left[d\left(x, A_{p q r}\right)\right]-3,\left[d\left(x, A_{p q r}\right)\right],\right\}
$$

and therefore $\left|\left[d\left(x, A_{m_{p} n_{q} k_{r}}\right)\right]-d\left(x, \tilde{A_{p q r}}\right)\right|=1.5$, for $(m, n, k) \geq\left(m_{*}, n_{*}, k_{*}\right)$ and $p \in(1,2,3, \ldots, u), q \in(1,2,3, \ldots, v)$ and $r \in(1,2,3, \ldots, w)$. Hence

$$
\left|\left[d\left(x, A_{m n k}\right)\right]-d(\tilde{x}, A)\right|= \begin{cases}1.5, & \text { if }|., .| \text { is the maximal matrix; } \\ 1.5 \sqrt{u v w}, & \text { if }|., .| \text { is the Euclidean metric. }\end{cases}
$$

for $m \geq m_{*}, n \geq n_{*}, k \geq k_{*}$. That means by definition $d(\tilde{x}, A) \in \operatorname{LIM}^{r}\left[d\left(x, A_{m n k}\right)\right]$ for $r= \begin{cases}1.5, & \text { if }|., .| \text { is the maximal matrix; } \\ 1.5 \sqrt{u v w}, & \text { if }|., .| \text { is the Euclidean metric. }\end{cases}$ 
Note. The Theorem 4 of parameters $r$ is optimal. Suppose one cannot give smaller parameters which are suitable for any case.

Example. $d\left(x, A_{m n k}\right)^{1}=d\left(x, A_{m n k}\right)^{2}=\ldots=d\left(x, A_{m n k}\right)^{u v w}=\frac{(-3)^{m n k}}{m n k}$. Then $d\left(x, A_{m n k}\right)=$ $\left[d\left(x, A_{m n k}\right)^{1}, d\left(x, A_{m n k}\right)^{2}, \ldots, d\left(x, A_{m n k}\right)^{u v w}\right] \rightarrow d(x, A)=0$ and

$d\left(x, A_{m n k}\right)= \begin{cases}0, & \text { if }(m, n, k) \text { is even; } \\ -3, & \text { if }(m, n, k) \text { is odd }\end{cases}$

Since $|-3-0|= \begin{cases}3, & \text { if }|., .| \text { is the maximal metric; } \\ \sqrt{u v w}, & \text { if }|., .| \text { is the Euclidean metric, }\end{cases}$

we have

$d(x, A) \notin \operatorname{LIM}^{r}\left[d\left(x, A_{m n k}\right)\right]$ for $r< \begin{cases}3, & \text { if }|., .| \text { is the maximal matrix; } \\ \sqrt{u v w}, & \text { if }|., .| \text { is the Euclidean metric. }\end{cases}$

and

$\operatorname{LIM}^{r}\left[d\left(x, A_{m n k}\right)\right]=\varnothing$ for $r< \begin{cases}1.5 & \text { if }|., .| \text { is the maximal matrix } \\ 1.5 \sqrt{u v w} & \text { if }|., .| \text { is the Euclidean metric. }\end{cases}$

Theorem 5. A Wijsman triple sequence $\left(d\left(x, A_{m n k}\right)\right) \rightarrow d(x, A) \Longleftrightarrow \operatorname{LIM}^{r} d\left(x, A_{m n k}\right)=$ $\bar{B}_{r}(d(x, A))$.

Proof. To prove that $\operatorname{LIM}^{r} d\left(x, A_{m n k}\right)=\bar{B}_{r}(d(x, A)) \Longrightarrow d\left(x, A_{m n k}\right) \rightarrow d(x, A)$.

Assume that contrary that the Wijsman triple sequence $\left(d\left(x, A_{m n k}\right)\right)$ has a cluster point $d(x, A)^{\prime}$ different from $d(x, A)$. Then the point

$$
\begin{gathered}
d\left(\bar{x}^{-}, A\right)=d(x, A)+\frac{r}{\left|d(x, A)-d(x, A)^{\prime}\right|}\left(d(x, A)-d(x, A)^{\prime}\right) \\
d\left(x^{-}, A\right)-d(x, A)^{\prime}=d(x, A)-d(x, A)^{\prime}+\frac{r\left[\left(d(x, A)-d(x, A)^{\prime}\right)-\left(d(x, A)^{\prime}-d(x, A)^{\prime}\right)\right]}{\left|d(x, A)-d(x, A)^{\prime}\right|} \\
\left|d\left(x^{-}, A\right)-d(x, A)^{\prime}\right|=\left|d(x, A)-d(x, A)^{\prime}\right|+\frac{r\left|d(x, A)-d(x, A)^{\prime}\right|}{\left|d(x, A)-d(x, A)^{\prime}\right|} \\
\left|d\left(x^{-}, A\right)-d(x, A)^{\prime}\right|=\left|d(x, A)-d(x, A)^{\prime}\right|+r>r .
\end{gathered}
$$

Since $d(x, A)^{\prime}$ is a cluster point, we have $d(\bar{x}, A) \notin \operatorname{LIM}^{r} d\left(x, A_{m n k}\right)$, a contradiction to $|d(\bar{x}, A)-d(x, A)|=r$ and $\operatorname{LIM}^{r} d\left(x, A_{m n k}\right)=\bar{B}_{r}(d(x, A))$. Thus $d(x, A)$ is the cluster point of the Wijsman triple sequence $\left(d\left(x, A_{m n k}\right)\right)$ as an analytic sequence in three dimensional metric space. Hence $\left(d\left(x, A_{m n k}\right)\right) \rightarrow d(x, A)$.

Theorem 6. $\operatorname{LIM}^{r}\left(d\left(x, A_{m n k}\right)\right)=\operatorname{LIM} \inf \bar{B}_{r}\left(d\left(x, A_{m n k}\right)\right)$.

Proof. Assume that $y \in \operatorname{LIM}^{r}\left(d\left(x, A_{m n k}\right)\right)$. Define then

$d\left(y, A_{m n k}\right)=\left\{\begin{array}{l}d\left(x, A_{m n k}\right)+\frac{r}{\left|d\left(y, A_{m n k}\right)-d\left(x, A_{m n k}\right)\right|}\left(d\left(y, A_{m n k}\right)-d\left(x, A_{m n k}\right)\right), \\ \text { if }\left|d\left(y, A_{m n k}\right)-d\left(x, A_{m n k}\right)\right|>r ; \\ d\left(y, A_{m n k}\right), \text { otherwise, }\end{array}\right.$

$d\left(y, A_{m n k}\right)-d\left(y, A_{m n k}\right)=$

$=\left\{\begin{array}{l}\left(d\left(x, A_{m n k}\right)-d\left(y, A_{m n k}\right)\right)+ \\ \frac{r}{\left|d\left(y, A_{m n k}\right)-d\left(x, A_{m n k}\right)\right|}\left[\left(d\left(y, A_{m n k}\right)-d\left(y, A_{m n k}\right)\right)-\left(d\left(x, A_{m n k}\right)-d\left(y, A_{m n k}\right)\right)\right], \\ \text { if }\left|d\left(y, A_{m n k}\right)-d\left(x, A_{m n k}\right)\right|>r ; \\ d\left(y, A_{m n k}\right)-d\left(y, A_{m n k}\right), \text { otherwise, }\end{array}\right.$ 
$\left|d\left(y, A_{m n k}\right)-d\left(y, A_{m n k}\right)\right|=$

$=\left\{\begin{array}{l}\left|d\left(x, A_{m n k}\right)-d\left(y, A_{m n k}\right)\right|-\frac{r}{\left|d\left(y, A_{m n k}\right)-d\left(x, A_{m n k}\right)\right|}\left|d\left(x, A_{m n k}\right)-d\left(y, A_{m n k}\right)\right|, \\ \text { if }\left|d\left(y, A_{m n k}\right)-d\left(x, A_{m n k}\right)\right|>r ; \\ 0, \text { otherwise, }\end{array}\right.$

$\left|d\left(y, A_{m n k}\right)-d\left(y, A_{m n k}\right)\right|=$

$=\left\{\begin{array}{l}\left|d\left(y, A_{m n k}\right)-d\left(x, A_{m n k}\right)\right|-\frac{r}{\left|d\left(y, A_{m n k}\right)-d\left(x, A_{m n k}\right)\right|}\left|d\left(y, A_{m n k}\right)-d\left(x, A_{m n k}\right)\right|, \\ \text { if }\left|d\left(y, A_{m n k}\right)-d\left(x, A_{m n k}\right)\right|>r, \\ 0, \text { otherwise, }\end{array}\right.$

$\left|d\left(x, A_{m n k}\right)-d\left(y, A_{m n k}\right)\right|=$

$\left\{\begin{array}{l}\left|d\left(y, A_{m n k}\right)-d\left(x, A_{m n k}\right)\right|-r, \quad \text { if }\left|d\left(y, A_{m n k}\right)-d\left(x, A_{m n k}\right)\right|>r ; \\ 0, \text { otherwise. }\end{array}\right.$

Therefore, $d\left(y, A_{m n k}\right) \in \operatorname{LIM}^{r}\left(d\left(x, A_{m n k}\right)\right)$ yields that $d\left(y, A_{m n k}\right) \rightarrow d\left(y, A_{m n k}\right)$ as $m, n, k \rightarrow \infty$. But $\left|d\left(x, A_{m n k}\right)-d\left(y, A_{m n k}\right)\right| \leq r$, i.e., $d\left(y, A_{m n k}\right) \in \bar{B}_{r}\left(d\left(x, A_{m n k}\right)\right)$. Consequently, $\lim _{m n k \rightarrow \infty} d\left(d\left(y, A_{m n k}\right), \bar{B}_{r}\left(d\left(x, A_{m n k}\right)\right)\right)=0$. Hence by definition we get

$$
d\left(y, A_{m n k \rightarrow \infty}\right) \in \operatorname{LIM} \inf \bar{B}_{r}\left(d\left(x, A_{m n k}\right)\right) .
$$

If $d\left(y, A_{m n k}\right) \in \operatorname{LIM} \inf \bar{B}_{r}\left(d\left(x, A_{m n k}\right)\right)$, then there exists a Wijsman triple sequence $\left(d\left(y, A_{m n k}\right)\right)$ such that $\left(d\left(y, A_{m n k}\right)\right) \rightarrow d\left(y, A_{m n k}\right)$ as $m, n, k \rightarrow \infty$ and $d\left(y, A_{m n k}\right) \in$ $\bar{B}_{r}\left(d\left(x, A_{m n k}\right)\right)$, i.e.,

$$
\left|d\left(x, A_{m n k}\right)-d\left(y, A_{m n k}\right)\right| \leq r \Longrightarrow d\left(y, A_{m n k}\right) \in \operatorname{LIM}^{r}\left(d\left(x, A_{m n k}\right)\right) .
$$

Definition 5. $F: \mathbb{R}_{+} \rightarrow 3^{X \times X \times X}$ with $F(r)=\operatorname{LIM}^{r} d\left(x, A_{m n k}\right), F$ is said to be Wijsman lower semi-continuous (l.s.c) of Wijsman triple sequence $\left(d\left(x, A_{m n k}\right)\right)$ at $r$, if for each open set $U$ satisfying $F(r) \bigcap U \neq \varnothing \exists$ a neighbourhood $V(r)$ such that $t \in V(r) \Longrightarrow F(t) \bigcap U \neq \varnothing$. It is called Wijsman upper semi-continuous (u.s.c) at $r$ if for each open set $U$ containing $F(r)$ there is a neigh bour hood $V(r)$ such that $t \in V(r) \Longrightarrow F(t) \subset U \neq \varnothing$. We say that if $F$ is l.s.c or u.s.c on $I$ then $r \in I$.

A Wijsman triple sequence $x=\left(x_{m n k}\right)$ is an dependence of $r$-limit $\operatorname{LIM}^{r} d\left(x, A_{m n k}\right)$ of a fixed Wijsman triple sequence $\left(x_{m n k}\right)$ on a varying parameter $r$, then

$$
\operatorname{LIM}^{r_{1}}\left(d\left(x, A_{m n k}\right)\right) \subseteq \operatorname{LIM}^{r_{2}}\left(d\left(x, A_{m n k}\right)\right) \text { as } r_{1}<r_{2} .
$$

Theorem 7. If $r \geq 0$ and $\sigma>0$ then

(i) $\operatorname{LIM}^{r}\left(d\left(x, A_{m n k}\right)\right)+\bar{B}_{\sigma}(0) \subseteq \operatorname{LIM}^{r+\sigma}\left(d\left(x, A_{m n k}\right)\right)$,

(ii) $\bar{B}_{\sigma}\left(d\left(y, A_{m n k}\right)\right) \subseteq \operatorname{LIM}^{r}\left(d\left(x, A_{m n k}\right)\right) \Longrightarrow d\left(y, A_{m n k}\right) \in \operatorname{LIM}^{r-\sigma} d\left(x, A_{m n k}\right)$.

Proof. (i) Let $d\left(y, A_{m n k}\right) \in \operatorname{LIM}^{r} d\left(x, A_{m n k}\right)$ and $z \in \bar{B}_{\sigma}(0)$, for all $\varepsilon>0 \exists$ an $m_{\varepsilon}, n_{\varepsilon}, k_{\varepsilon}$ such that

$$
\left|d\left(x, A_{m n k}\right)-d\left(y, A_{m n k}\right)\right|<r+\varepsilon \text { if } m \geq m_{\varepsilon}, n \geq n_{\varepsilon}, k \geq k_{\varepsilon}
$$

$\Longrightarrow|z| \leq \sigma \Longrightarrow\left|d\left(x, A_{m n k}\right)-(y+z)\right|<r+\sigma+\varepsilon$ for all $m \geq m_{\varepsilon}, n \geq n_{\varepsilon}, k \geq k_{\varepsilon}$.

Hence $y+z \in \operatorname{LIM}^{r+\sigma} d\left(x, A_{m n k}\right)$.

(ii) Let $c$ be an arbitrary cluster point of $\left(d\left(x, A_{m n k}\right)\right)$. If $\left|d\left(y, A_{m n k}\right)-c\right|>r-\sigma$ then

$$
d(x, A)=d\left(y, A_{m n k}\right)+\frac{\sigma}{\left|d\left(y, A_{m n k}\right)-c\right|}\left(d\left(y, A_{m n k}\right)-c\right),
$$




$$
\begin{gathered}
d(x, A)-c=\left(d\left(y, A_{m n k}\right)-c\right)+\frac{\sigma}{\left|d\left(y, A_{m n k}\right)-c\right|}\left[\left(d\left(y, A_{m n k}\right)-c\right)-(c-c)\right], \\
|d(x, A)-c|=|d(x, A)-c|+\frac{\sigma}{|d(x, A)-c|}|d(x, A)-c|, \\
|d(x, A)-c|=\left|d\left(y, A_{m n k}\right)-c\right|+\sigma<r-\sigma+\sigma=r .
\end{gathered}
$$

Since $c$ is a cluster point of the Wijsman triple sequence $\left(d\left(x, A_{m n k}\right)\right)$ then

$$
\operatorname{LIM}^{r}\left(d\left(x, A_{m n k}\right)\right) \subseteq \bar{B}_{r}(c),
$$

we have $d(x, A) \notin \operatorname{LIM}^{r}\left(d\left(x, A_{m n k}\right)\right)$, a contradiction to $\left|d(x, A)-d\left(x, y_{m n k}\right)\right|=\sigma$ and $\bar{B}_{\sigma}\left(d\left(y, A_{m n k}\right)\right) \subseteq \operatorname{LIM}^{r}\left(d\left(x, A_{m n k}\right)\right)$. Hence $\left|d\left(y, A_{m n k}\right)-c\right| \leq r-\sigma \forall c \in C$. Consequently, it follows $d\left(y, A_{m n k}\right) \in \bigcap_{c \in C} \bar{B}_{r-\sigma}(c)=\operatorname{LIM}^{r-\sigma} d\left(x, A_{m n k}\right)$. In general,

$$
\operatorname{LIM}^{r}\left(d\left(x, A_{m n k}\right)\right)+\bar{B}_{\sigma}(0) \neq \operatorname{LIM}^{r+\sigma}\left(d\left(x, A_{m n k}\right)\right) .
$$

For instance, the Wijsman triple sequence $\left(x_{m n k}\right)$ in the three dimensional Euclidean space with

$$
d\left(x, A_{m n k}\right)=\left(0, \xi_{m n k}\right) \in \mathbb{R}^{3}, \text { where } \xi_{m n k}=(-1)^{m+n+k}, m, n, k=1,2,3, \cdots
$$

satisfies, if $r=0.5$ and $\sigma=0.5$ then

$$
\operatorname{LIM}^{0.5}\left(d\left(x, A_{m n k}\right)\right)+\bar{B}_{0.5}(0)=\varnothing+\bar{B}_{0.5}(0)=\varnothing \neq\{(0,0)\}=\operatorname{LIM}^{1}\left(d\left(x, A_{m n k}\right)\right) .
$$

Now consider if $r=1$ and $\sigma=1$ then

$$
\operatorname{LIM}^{1}\left(d\left(x, A_{m n k}\right)\right)+\bar{B}_{1}(0)=\{(0,0)\}+\bar{B}_{1}(0)=\bar{B}_{1}(0)=\operatorname{LIM}^{2}\left(d\left(x, A_{m n k}\right)\right),
$$

while

$$
\begin{gathered}
\left|(\sqrt{2}, 0)-d\left(x, A_{m n k}\right)\right|=|(\sqrt{2}, \pm 1)|=2.414 \approx 2 \text { for all } m, n, k \text { implies } \\
(\sqrt{2}, 0) \in \operatorname{LIM}^{2} \backslash \bar{B}_{1}(0) \text {, i.e. }
\end{gathered}
$$

$\Longrightarrow \operatorname{LIM}^{1}+\bar{B}_{1}(0) \neq \operatorname{LIM}^{2}\left(d\left(x, A_{m n k}\right)\right)$. Similarly, consider if $r=2$ and $\sigma=1$ then

$$
\operatorname{LIM}^{2}\left(d\left(x, A_{m n k}\right)\right)+\bar{B}_{1}(0)=\{(\sqrt{2}, 0)\}+\bar{B}_{1}(0)=\operatorname{LIM}^{3}\left(d\left(x, A_{m n k}\right)\right),
$$

while

$$
\begin{gathered}
\left|(\sqrt{11}, 0)-d\left(x, A_{m n k}\right)\right|=|(\sqrt{11}, \pm 1)|=3.23 \approx 3 \text { for all } m, n, k \text { implies } \\
(\sqrt{11}, 0) \in \operatorname{LIM}^{3} \backslash \bar{B}_{1}(0), \text { i.e. }
\end{gathered}
$$

$\Longrightarrow \operatorname{LIM}^{2}+\bar{B}_{1}(0) \neq \operatorname{LIM}^{3}\left(d\left(x, A_{m n k}\right)\right)$, and so on.

Define :

$$
\bar{r}=\inf \left\{r \in \mathbb{R}^{+}: \operatorname{LIM}^{r}\left(d\left(x, A_{m n k}\right)\right) \neq \varnothing\right\} .
$$

By monotonicity we have

$$
\operatorname{LIM}^{r}\left(d\left(x, A_{m n k}\right)\right)\left\{\begin{array}{l}
=\varnothing \text { for } r<\bar{r} \\
\neq \varnothing \text { for } r>\bar{r}
\end{array}\right.
$$

for all $r>\bar{r}$ and $\sigma \in(0, r-\bar{r}), \operatorname{LIM}^{r}\left(d\left(x, A_{m n k}\right)\right)$ always contains some ball with radius $\sigma$, that means at least

$$
\operatorname{int}\left(\operatorname{LIM}^{r}\left(d\left(x, A_{m n k}\right)\right)\right) \neq \varnothing \text { for } r>\bar{r}
$$


Hence

$$
\operatorname{int}\left(\operatorname{LIM}^{r}\left(d\left(x, A_{m n k}\right)\right)\right)=\varnothing \Longrightarrow r \leq \bar{r} \text { and } \stackrel{\operatorname{LIM}^{\prime}}{\operatorname{LI}}\left(d\left(x, A_{m n k}\right)\right)=\varnothing \text { for } r^{\prime} \in[0, r)
$$

Theorem 8. $c l\left(\bigcup_{0 \leq r^{\prime}<r} \operatorname{LIM}^{r^{\prime}}\left(d\left(x, A_{m n k}\right)\right)\right) \subseteq \operatorname{LIM}^{r}\left(d\left(x, A_{m n k}\right)\right)=\bigcap_{r^{\prime}>r} \operatorname{LIM}^{r^{\prime}}$ $\left(d\left(x, A_{m n k}\right)\right)$. If $r \neq \bar{r}$ then $c l\left(\bigcup_{0 \leq r^{\prime}<r} \operatorname{LIM}^{r^{\prime}} d\left(x, A_{m n k}\right)\right)=\operatorname{LIM}^{r} d\left(x, A_{m n k}\right)$.

Proof. By the the condition of monotonicity in Theorem 8 and the closedness of $r$-limit we have $c l\left(\bigcup_{0 \leq r^{\prime}<r} \operatorname{LIM}^{r^{\prime}}\left(d\left(x, A_{m n k}\right)\right)\right) \subseteq \operatorname{LIM}^{r}\left(d\left(x, A_{m n k}\right)\right) \subseteq \bigcap_{r^{\prime}>r} \operatorname{LIM}^{r^{\prime}}\left(d\left(x, A_{m n k}\right)\right)$.

Consider an arbitrary $d\left(y, A_{m n k}\right) \in X \backslash \operatorname{LIM}^{r}\left(d\left(x, A_{m n k}\right)\right)$ there is an $\varepsilon>0$ such that $\forall(i, j, \ell) \in \mathbb{N}^{3} \exists m \geq i, n \geq j, k \geq \ell:\left|d\left(x, A_{m n k}\right)-d\left(y, A_{m n k}\right)\right| \geq r+\varepsilon$.

$\Longrightarrow$ for $r^{\prime}<r+\varepsilon$ that $\varepsilon^{\prime}=r+\varepsilon-r^{\prime}>0$ and $\forall(i, j, \ell) \in \mathbb{N}^{3} \exists m \geq i, n \geq j, k \geq \ell$ : $\left|d\left(x, A_{m n k}\right)-d\left(y, A_{m n k}\right)\right| \geq r^{\prime}+\varepsilon^{\prime}$.

Thus $d\left(y, A_{m n k}\right) \notin \operatorname{LIM}^{r^{\prime}}\left(d\left(x, A_{m n k}\right)\right)$ for $r^{\prime}<r+\varepsilon \Longrightarrow y \notin \bigcap_{r^{\prime}>r} \operatorname{LIM}^{r^{\prime}} x_{m n k}$. Hence

$$
\operatorname{LIM}^{r}\left(d\left(x, A_{m n k}\right)\right)=\bigcap_{r^{\prime}>r} \operatorname{LIM}^{r^{\prime}}\left(d\left(x, A_{m n k}\right)\right) .
$$

For $r<\bar{r}$, it is clear that

$$
c l\left(\bigcup_{0 \leq r^{\prime}<r} \operatorname{LIM}^{r^{\prime}}\left(d\left(x, A_{m n k}\right)\right)\right)=\operatorname{LIM}^{r}\left(d\left(x, A_{m n k}\right)\right)=\varnothing .
$$

Let $r=r_{1}>\bar{r}$ and $r_{0}=\frac{\bar{r}+r_{1}}{2}$. Since $r_{0}>\bar{r}$, we can choose a $d\left(y_{1}, A_{m n k}\right) \in \mathrm{LIM}^{r_{0}}$ $\left(d\left(x, A_{m n k}\right)\right) \neq \varnothing$. For an arbitrary $y_{2} \in \operatorname{LIM}^{r_{1}}\left(d\left(x, A_{m n k}\right)\right)$ and $\lambda \in[0,1]$ we get

$$
\begin{gathered}
d\left(y_{\lambda}, A_{m n k}\right)=(1-\lambda) d\left(y_{1}, A_{m n k}\right)+ \\
+\lambda d\left(y_{2}, A_{m n k}\right) \in \operatorname{LIM}^{(1-\lambda)}\left(d\left(y_{1}, A_{m n k}\right)+\lambda d\left(y_{2}, A_{m n k}\right) d\left(x, A_{m n k}\right)\right) .
\end{gathered}
$$

Consequently, $d\left(y_{\lambda}, A_{m n k}\right) \in \bigcup_{0 \leq r^{\prime}<r} \operatorname{LIM}^{r^{\prime}}\left(d\left(x, A_{m n k}\right)\right)$ for $\lambda \in[0,1)$. Since

$$
d\left(y_{\lambda}, A_{m n k}\right)=(1-\lambda) d\left(y_{1}, A_{m n k}\right)+\lambda d\left(y_{2}, A_{m n k}\right),
$$

then

$$
\begin{gathered}
\left(d\left(y_{\lambda}, A_{m n k}\right)-d\left(y_{2}, A_{m n k}\right)\right)=(1-\lambda)\left(d\left(y_{1}, A_{m n k}\right)-d\left(y_{2}, A_{m n k}\right)\right) \\
\text { i.e., }\left|d\left(y_{\lambda}, A_{m n k}\right)-d\left(y_{2}, A_{m n k}\right)\right|=(1-\lambda)\left|d\left(y_{1}, A_{m n k}\right)-d\left(y_{2}, A_{m n k}\right)\right| \rightarrow 0 \text { as } \lambda \rightarrow 1,
\end{gathered}
$$

it follows $d\left(y_{2}, A_{m n k}\right) \in \operatorname{cl}\left(\bigcup_{0 \leq r^{\prime}<r} \operatorname{LIM}^{r^{\prime}} d\left(x, A_{m n k}\right)\right)$. Hence

$$
c l\left(\bigcup_{0 \leq r^{\prime}<r} \operatorname{LIM}^{r^{\prime}}\left(d\left(x, A_{m n k}\right)\right)\right)=\operatorname{LIM}^{r}\left(d\left(x, A_{m n k}\right)\right) \text { holds for } r>\bar{r} \text {. }
$$

3. Acknowledgement. The authors are thankful to the anonymous Reviewers for their valuable suggestions and constructive comments. 


\section{REFERENCES}

1. S. Aytar, Rough statistical convergence, Numer. Funct. Anal. Optimiz, 29 (2008), №3-4, 291-303.

2. S. Aytar, The rough limit set and the core of a real sequence, Numer. Funct. Anal. Optimiz, 29 (2008), №3-4, 283-290.

3. A. Esi, On some triple almost lacunary sequence spaces defined by Orlicz functions, Research and Reviews: Discrete Mathematical Structures, 1 (2014), №2, 16-25.

4. A. Esi, M. Necdet Catalbas, Almost convergence of triple sequences, Global J. Math. Anal., 2 (2014), №1, 6-10.

5. A. Esi, E. Savas, On lacunary statistically convergent triple sequences in probabilistic normed space, Appl. Math. Inf. Sci., 9 (2015), №5, 2529-2534.

6. A.J. Datta, A. Esi, B.C. Tripathy, Statistically convergent triple sequence spaces defined by Orlicz function, J. Math. Anal., 4 (2013), №2, 16-22.

7. S. Debnath, B. Sarma, B.C. Das, Some generalized triple sequence spaces of real numbers, J. Nonlinear Anal Optimiz, 6 (2015), №1, 71-79.

8. E. Dündar, C. Cakan, Rough I- convergence, Demonstratio Mathematica, accepted.

9. H.X. Phu, Rough convergence in normed linear spaces , Numer. Funct. Anal. Optimiz, 22 (2001), 199222.

10. H.X. Phu, Rough continuity of linear operators, Numer. Funct. Anal. Optimiz, 23, (2002), 139-146.

11. H.X. Phu, Rough convergence in infinite dimensional normed spaces, Numer. Funct. Anal. Optimiz, 24, (2003), 285-301.

12. A. Sahiner, M. Gurdal, F.K. Duden, Triple sequences and their statistical convergence, Selcuk J. Appl. Math., 8 (2007), №2, 49-55.

13. A. Sahiner, B.C. Tripathy, Some I related properties of triple sequences, Selcuk J. Appl. Math., 9 (2008), №2, 9-18.

14. N. Subramanian, A. Esi, The generalized tripled difference of $\chi^{3}$ sequence spaces, Global J. Math. Anal., 3 (2015), №2, 54-60.

Department of Mathematics, SASTRA University

Thanjavur, India

nsmaths@yahoo.com

Department of Mathematics

Adiyaman University, Turkey

aesi23@hotmail.com 